\title{
Retention Rates of ACLS Skills in Senior Medical Students
}

\author{
Onedera $\mathrm{T}^{1}$, Nguyen $\mathrm{M}^{2}$, Abrahamsen $\mathrm{N}^{3}$, Schocken $\mathrm{D}^{4}$, Peckler $\mathrm{B}^{1, *}$ \\ ${ }^{I}$ FACEP FACEM University of South Florida, Department of Emergency Medicine, USA \\ ${ }^{2}$ Department of Emergency Medicine, Wellington Regional Hospital, Wellington, New Zealand \\ ${ }^{3}$ University of South Florida, Department of Emergency Medicine Nadia, USA \\ ${ }^{4}$ University of South Florida, Director of Clinical Skills Centre, USA
}

\begin{abstract}
Objectives: The aim of this study was to assess the retention rate of Advanced Cardiac Life Support (ACLS) skills in third-year medical students who had few clinical opportunities or responsibilities to use them. It was hypothesised that using high-fidelity simulation would increase skills retention.

Methods: This was a prospective observational study of 120 rising third year medical students. All students took and eventually passed a full two-day ACLS provider course. Subsequently, during the end of the year Comprehensive Clinical Performance Examination (CCPX) all students participated in a mega code with a Laerdal Hi-Fidelity Simulator. Their performance in 25 critical actions was dichotomously rated as "successful" or "not successful."

Results: All students passed the initial ACLS course, six required standard remediation. One hundred and sixteen students took the end of year CCPX exam, and a success rate for critical actions ranged between 3.4\% and 100\%. Most students did well at recognition of fatal arrhythmias and cardiopulmonary resuscitation (CPR). Students performed poorly in airway/breathing assessment and management, as well as management of arrhythmias.

Conclusions: ACLS skills are an important skill set for up coming physicians, and unless they are used in practice, they deteriote very quickly. Simulation did not increase student retention rates of ACLS skills to a great extent. Students did well in identifying problems, but did not treat these appropriately. This study suggests that educators should examine the goals of teaching ACLS to providers who have not clearly defined role in resuscitation.
\end{abstract}

Keywords: ACLS retention, simulation, medical students.

\section{INTRODUCTION}

American Heart Association, since their first publishing in 1974, has provided health care professionals the Advanced Cardiac Life Support (ACLS) course as a standardized guideline for assessment and treatment of patients with acute cardiac conditions. ACLS providers need to be re-certified every two years as the ability to retain any skill is based upon repetition and use. This study attempted to evaluate the ability of rising third year medical students who had very little clinical experience or responsibilities to maintain their ACLS knowledge and skills.

ACLS retention has been studied on physicians in training and simulation is of added educational benefit [1]. Wayne DB et al. studied the retention rate of ACLS skills in internal medicine residents. Their prospective cohort study revealed that ACLS skills were retained for 14 months with deliberate practice sessions [2]. Research supports that simulation-based ACLS courses provide better results than

*Address correspondence to these authors at the FACEP FACEM University of South Florida, Department of Emergency Medicine, USA;

Tel: 813-627-5931; Fax: 813-254-6440; E-mail: bpeckler@health.usf.edu textbook-based ones, including a higher adherence to the AHA guidelines [3] and better performance at the mega code testing two months after the course [4]. It is unclear why physicians (residents and fellows) maintain their ACLS skills better than nurses. Medical students have not assumed the role or responsibilities of a physician and are not qualified as nurses or paramedics. Their ability to retain ACLS skills may be more difficult because they have no clear role in a hospital setting, they may be asked to help, however have no predefined role.

There are few articles examining the ACLS retention rate in medical students. This may be based on the fact that while ACLS courses are commonly provided to medical students, assessment of the student's retention is not often done. The third year of medical school is the first intense clinical exposure they experience. They are part of the clinical teams yet they have few responsibilities. Medical students may witness a cardiac arrest during clinical rotations and occasionally, they may be asked to assist in resuscitation tasks. Students are not regularly assigned an essential role on the code teams, and therefore they're not required to be certified in ACLS for their clinical rotations. ACLS is, however, a core concept of cardiopulmonary resuscitation, and therefore needs to be a part of their curriculum. 

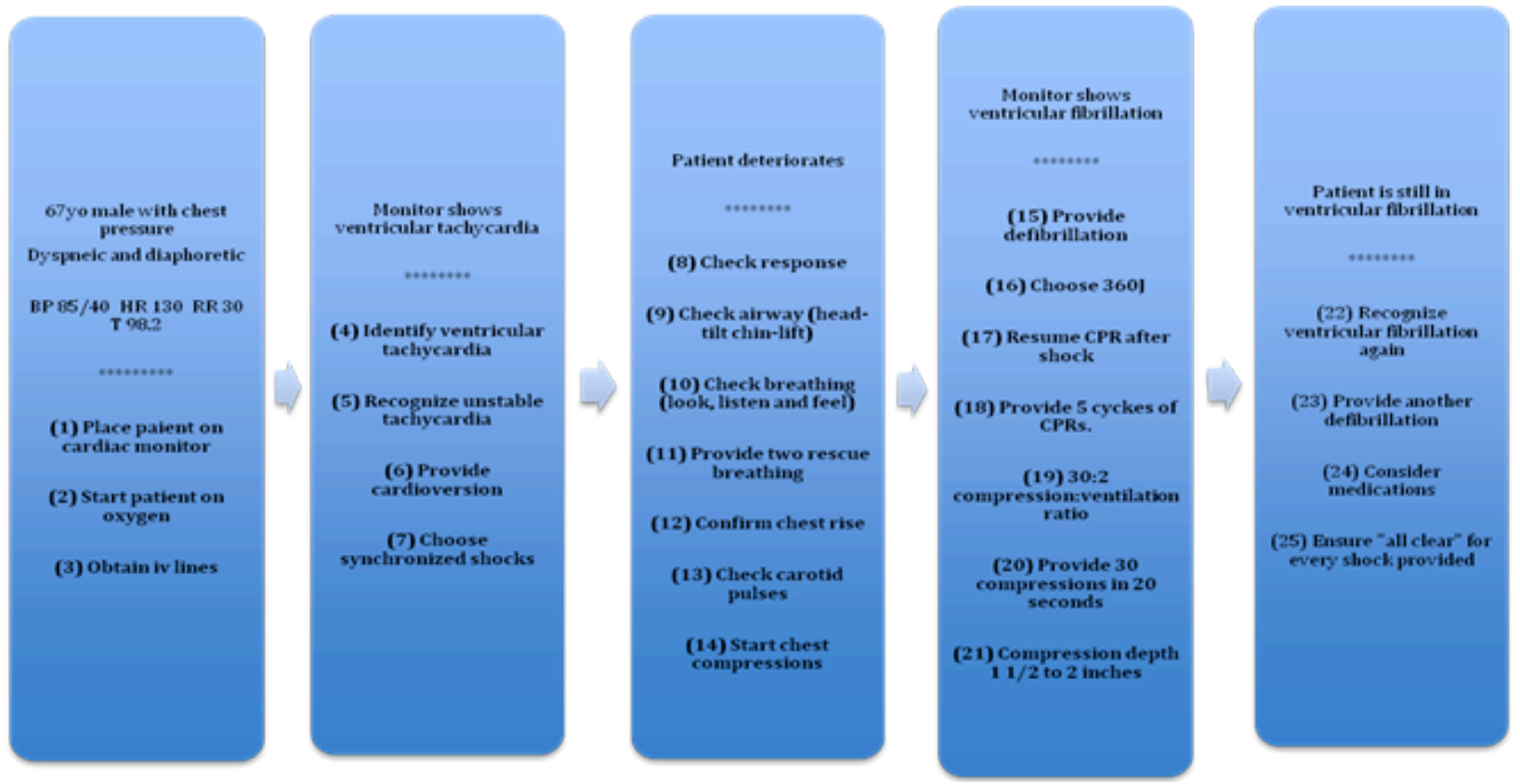

Fig. (1). Case algorithm, Edward Cardia.

This study sought to evaluate the retention rates of ACLS knowledge and skills. Testing ACLS skills retention in third year medical students is unique in that students rotate through various clinical departments and have different clinical experiences. Additionally, the field of medicine that the student intends to pursue may have an impact upon a student's interest and retention of the ACLS protocols. The hypothesis of this study was the use of hi-fidelity simulation in teaching ACLS would demonstrate a higher rate of retention. Data from medical students could provide important insights into ACLS retention, including protocols well remembered and ones not. The consequences of this study could advocate for additional ACLS protocols to reinforce future curriculum development.

\section{METHODS}

This was a prospective observational study. We obtained exempt certification from the Institutional Review Board. All the students were informed that identification would be redacted and no individual would be identified, or adversely affected by the study. Additionally, no grades would be affected by the results of the study. Both verbal and signed consent was obtained for this study.

All rising $3^{\text {rd }}$ year medical students $(\mathrm{N}=120)$ took a full two-day ACLS provider course. All students successfully passed the written tests and the mega code scenarios at the end of the course. Remediation was required for six students. During the end of year CCPX final exam, the students were put into a scenario where they were the code team leader. This was a non-graded station in this exam activity. The scenario consisted of managing an unstable elderly patient with chest pain (a Laerdal Hi-Fidelity Simulator). The scenario included several confederate actors in the room. An ACLS instructor, running the simulator, served as evaluator, two ACLS certified nurses also were confederates in the room to assist as members of the code team.
Fig. (1) shows the flow of the mega code case. Mr. Edward Cardia (patient) was presented as a 67 year old male with chest pain, dyspnea and diaphoresis. Vital Signs: BP $85 / 40 \mathrm{mmHg}$, HR 130/min, RR 30/min and $\mathrm{T} 98.2 \mathrm{~F}$. Students were expected to place the patient on a cardiac monitor, start oxygen and obtain intravenous access. The patient complained of pain and was in distress and unstable. The evaluator read the monitor aloud, which showed all vital signs and the cardiac rhythm. Students needed to identify the ventricular tachycardia on the monitor, and recognize the unstable tachycardia from the clinical picture and vital signs. The management plan included cardioversion after choosing the synchronized mode. The patient's condition continued to deteriorate, prompting a reassessment. The protocol called for a responsiveness check, opening of airway and beginning of CPR, following recognition of no spontaneous breathing. The confederate actors could be assigned to these roles with verbal identification from student. Subsequently, an assessment of the carotid pulse (which demonstrated as absent) would then prompt initiation of chest compressions. The rhythm on the monitor changed to ventricular fibrillation. Students were expected to both recognize the rhythm and provide immediate defibrillation at $360 \mathrm{~J}$. Following shock, the students were to resume CPR. After 5 cycles of CPR, it was expected the student would check the monitor and pulse (which demonstrated continued ventricular fibrillation). Following a second defibrillation, and assessment, the students were to consider medication (epinephrine). A mark was checked each time the student asked for "all clear" prior to using the defibrillator.

\section{RESULTS}

One-hundred twenty third-year medical students participated in the ACLS course at the beginning of this study. All 120 students successfully completed the ACLS course and their competency for all the ACLS skills was $100 \%$ at the end of the ACLS course. Of the 120 , only 116 
Table 1. All Major Actions were Checked Dichotomously and Accounted for: "Successfully Performed or not"

\begin{tabular}{|c|c|c|c|}
\hline & Actions & \multicolumn{2}{|c|}{ Performance } \\
\hline 1 & Place monitor leads in proper position & Yes & No \\
\hline 2 & Provide oxygen & Yes & No \\
\hline 3 & Start intravenous lines & Yes & No \\
\hline 4 & Identify ventricular tachycardia & Yes & No \\
\hline 5 & Recognize unstable tachycardia & Yes & No \\
\hline 6 & Perform immediate cardioversion & Yes & No \\
\hline 7 & Choose synchronized shocks for cardioversion & Yes & No \\
\hline 8 & Check the response of the patient & Yes & No \\
\hline 9 & Open the airway using head-tilt chin-lift maneuver & Yes & No \\
\hline 10 & Look, listen and feel breathing & Yes & No \\
\hline 11 & Provide two rescue breathing using an ambu bag & Yes & No \\
\hline 12 & Confirm chest rise with an ambu bag breathing & Yes & No \\
\hline 13 & Check carotid pulses & Yes & No \\
\hline 14 & Perform immediate chest compressions & Yes & No \\
\hline 15 & Recognize ventricular fibrillation and defibrillate the patient & Yes & No \\
\hline 16 & Defibrillate at $360 \mathrm{~J}$ & Yes & No \\
\hline 17 & Resume CPR immediately after defibrillation & Yes & No \\
\hline 18 & Provide 5 cycles of CPR & Yes & No \\
\hline 19 & Provide ventilation/compression at $30: 2$ ratio & Yes & No \\
\hline 20 & Provide 30 compressions within 20 seconds & Yes & No \\
\hline 21 & Provide compressions 1.5 to 2 inches deep & Yes & No \\
\hline 22 & Identify ventricular fibrillation again & Yes & No \\
\hline 23 & Defibrillate the patient again & Yes & No \\
\hline 24 & Provide medications & Yes & No \\
\hline 25 & Ensure "everyone is clear" before defibrillation & Yes & No \\
\hline
\end{tabular}

Table 2. Result

\begin{tabular}{|c|c|c|c|}
\hline Actions & Yes $(N=116)$ & Rate (\%) & $+/-($ for $95 \% C I)$ \\
\hline Place monitor leads in proper position & 116 & 100.0 & 0.0 \\
\hline Start intravenous lines & 77 & 66.4 & 8.6 \\
\hline Identify ventricular tachycardia & 104 & 89.7 & 5.5 \\
\hline Perform immediate cardioversion & 66 & 56.9 & 9.0 \\
\hline Choose synchronized shocks for cardioversion & 37 & 31.9 & 8.5 \\
\hline
\end{tabular}


Table 2. contd..

\begin{tabular}{|c|c|c|c|}
\hline Actions & Yes $(N=116)$ & Rate (\%) & $+/-($ for $95 \% \mathrm{CI})$ \\
\hline Check the response of the patient & 27 & 23.3 & 7.7 \\
\hline Open the airway using head-tilt chin-lift maneuver & 35 & 30.2 & 8.4 \\
\hline Look, listen and feel breathing & 61 & 52.6 & 9.1 \\
\hline Provide two rescue breathing using an ambu bag & 114 & 98.3 & 2.4 \\
\hline Confirm chest rise with an ambu bag breathing & 65 & 56.0 & 9.0 \\
\hline Check carotid pulses & 75 & 64.7 & 8.7 \\
\hline Perform immediate chest compressions & 113 & 97.4 & 2.9 \\
\hline Recognize ventricular fibrillation and defibrillate the patient & 81 & 69.8 & 8.4 \\
\hline Defibrillate at $360 \mathrm{~J}$ & 69 & 59.5 & 8.9 \\
\hline Resume CPR immediately after defibrillation & 76 & 65.5 & 8.6 \\
\hline Provide 5 cycles of CPR & 89 & 76.7 & 7.7 \\
\hline Provide ventilation/compression at 30:2 ratio & 113 & 97.4 & 2.9 \\
\hline Provide 30 compressions within 20 seconds & 105 & 90.5 & 5.3 \\
\hline Provide compressions 1.5 to 2 inches deep & 92 & 79.3 & 7.4 \\
\hline Identify ventricular fibrillation again & 98 & 84.5 & 6.6 \\
\hline Defibrillate the patient again & 89 & 76.7 & 7.7 \\
\hline Provide medications & 81 & 69.8 & 8.4 \\
\hline Ensure "everyone is clear" before defibrillation & 4 & 3.4 & 3.3 \\
\hline
\end{tabular}

students took the end of the year CCPX exam. The results, in chronological order, are presented in Table 2 . We utilized normal approximation to calculate the $95 \%$ CI of the proportions.

$p \pm Z_{C} \sqrt{\frac{P(1-P)}{n}}$

\section{DISCUSSION}

Most students retained the "Circulation" part of Basic Life Support while they did poorly at "Airway" and "Breathing." Only $55.2 \%$ of students provided oxygen to the patient. Very few students $(23.3 \%)$ checked the response of the patient and $30.2 \%$ of students were able to demonstrate the maneuver to open the airway. Nearly $99 \%$ (98.3\%) of students demonstrated successfully providing two rescue breaths with a bag valve mask, but observation of the videos tells us that the testing nurse, expecting the next move of the students, automatically passed an Ambu bag to the students without request. Performance of chest compression, though it involved many components (frequency and depth), was one-dimensional and therefore readily performed by the students. However, the assessment of airway and breathing involves a three-dimensional understanding of the respiratory system and requires multiple actions. The skill sets of airway/breathing assessment and management deteriote very quickly. Some BLS skills had much lower scores such as checking the response of the patient, checking pulses, chin lift maneuver, and basic breathing evaluation. Many students did better on the advanced skills and seemed to neglect the basic skills.

All students placed the patient on cardiac monitor $(100.0 \%)$ and most subsequently identified fatal arrhythmias such as ventricular tachycardia $(89.7 \%)$ and ventricular fibrillation $(84.5 \%)$ but a large percentage of students had difficulty in managing these rhythms and determining stable or unstable. The advance skills outlier was giving the "everyone clear" statement before defibrillation at 3.4\%. This may have been due to it not being emphasized during the original training course, understanding of the potential danger of electricity, or that it was a simulated scenario and they did not think it a danger.

This study is consistent with many other studies that showed skill degradation over time without interval practice [5-13]. This may explain why these third year students who are not specifically doing these skills year scored so low on some tasks. Brennen4 demonstrated similar poor success rates to ours in the lay public. It is clear that interval training increases retention [1,14-16]. Most studies did not look at specific skills within ACLS. One study however looked at a half-day "How to Save a Life" course to medical students at orientation before starting clinical rotations at the beginning of the third-year [17]. The students were assessed for their knowledge and skill retention during their $4^{\text {th }}$ year EM rotations. Retention rates were great for CPR $(97.1 \%)$, but they did less well for use of bag-valve mask $(82.4 \%)$, 
opening and maintaining airway (79.4\%), use of AED (73.5\%), use of airway adjuncts $(70.6 \%)$, treatment of pediatric choking victim $(64.7 \%)$, treatment of arrhythmias $(61.8 \%)$, and identification of arrhythmias (58.8\%). This was consistent with our findings.

We do not think that their poor performance was because of lack of knowledge, but rather in applying it. A highfidelity simulation mannequin, much like an actual patient, seemed to cause difficulty in recall. Students had difficulty utilizing their one-dimensional didactic knowledge as a decision tool for managing the actual situation exhibited by the high-fidelity simulation. The large variation in results may be due to different exposures that have had during the year and any experience in using the skills. At the time of the year when the test is given students are deciding on different career choices. It is understandable that a student who chooses a field of medicne that deals with critically ill patients would have more motivation to memorize the algorithms than a doctor who chooses a field of medicine that does not deal with acute emergencies. Actions cannot take place without both the knowledge and the bridge converting the knowledge into the action. This bridge between knowledge and actions decay very quickly. It is suggested that regular re-training intervals, using simulation, would help maintain these critical skills.

\section{CONCLUSION}

It was not surprising that students with little actual emergent clinical experience would have difficulties managing the more complex aspects of ACLS. Airway management and determining the stability of a patient are some of the cornerstones of resuscitation, but also some of the more complex aspects. Unless a practitioner has routine exposure to acute clinical situations, such as cardiac arrest, ACLS skills can quickly deteriorate over time. Students did well identifying the problem, but not in following the necessary treatment protocols. This study suggests that ACLS skills deteriorate over time without deliberate practice or use.

\section{CONFLICT OF INTEREST}

The authors confirm that this article content has no conflicts of interest.

\section{ACKNOWLEDGEMENTS}

Declared none.

\section{REFERENCES}

[1] Wayne DB, Butter J, Siddall VJ, et al.Mastery learning of advanced cardiac life support skills by internal medicine residents using simulation technology and deliberate practice. J Gen Intern Med 2006; 21(3): 251-6.

[2] Wayne DB, Siddall VJ, Butter J, et al. A longitudinal study of internal medicine residents' retention of advanced cardiac life support skills. Acad Med 2006; 81(10): S9-12.

[3] Wayne DB, Didwania A, Feinglass J, Fudala MJ, Barsuk JH, McGaghie WC. Simulation-based education improves quality of care during cardiac arrest team responses at an academic teaching hospital. Chest 2008; 133(1): 56-61.

[4] Schwid H, Rooke GA, Brian R, Sivarajan M. Use of a computerized advanced cardiac life support simulator improves retention of advanced cardiac life support guidelines better than a textbook review. Crit Care Med 1999; 27(4): 821-4.

[5] Brennan RT, Braslow A. Skill mastery in public CPR classess. Am J Emerg Med 1998; 16: 653-7.

[6] Wollard M, Whitfeild R, Smith A, et al. Skill acquisition and retention in automated external defribrillator (AED) use and CPR by lay responders: a prospective study. Resuscitation 2004; 60: 17-28.

[7] Kaye W, Mancini ME, Rallis SF. Advanced cardiac life support refresher course using standardized objective-based mega code testing. Crit Care Med 1987; 15: 55-60.

[8] O'Steen DS, Kee CC, Minick MP. The retention of advanced cardiac life support knowledge among registered nurses. J Nurs Staff Dev 1996; 12: 66-72.

[9] Moser DK, Dracup K, Guzy PM, Taylor SE, Breu C. Cardiopulmonary resuscitation skills retention in family members of cardiac patients. Am J Emer Med 1990; 8: 498-503.

[10] Donnelly P, Assar, Lester C. A comparison of manikin CPR performance by lay persons trained in three variations of basic life support guidelines. Resuscitation 2000; 45: 195-9.

[11] Chamberlain D, Smith A, Woollard M, et al. Trials of teaching methods in basic life support (3): comparison of simulated CPR performance after first training and at 6 months, with a note on the value of retraining. Resuscitation 2002; 53: 179-87.

[12] Anthonypillai F. Retention of advanced cardiopulmonary resuscitation knowledge by intensive care trained nurses. Intensive Crit Care Nurs 1992; 8: 180-4.

[13] Kaye W, Mancini ME. Retention of cardiopulmonary resuscitation skills by physicians, registered nurses, and the general public. Crit Care Med 1986; 14: 620-2.

[14] Ko PY. Comparison of a modified longitudinal simulation-based advanced cardiovascular life support to a traditional advanced cardiovascular life support curriculum in third-year medical students. Teach Learn Med 2011; 23(4): 324-30.

[15] Wayne DB, Butter J, Siddall VJ, et al. Mastery learning of advanced cardiac life support skills by internal medicine residents using simulation technology and deliberate practice. J Gen Intern Med 2006; 21(3): 251-6.

[16] Lo BM Comparison of traditional versus high-fidelity simulation in the retention of ACLS knowledge. Resuscitation 2011; 82(11): 1440-3

[17] Ander DS, Heilpern K, Goertz F, Click L, Kahn S. Effectiveness of a simulation-based medical student course on managing lifethreatening medical conditions. Simul Healthcare 2009; 4(4): 20711 . 